\title{
Analysis of fuel tank collision structure based on defense point method
}

Wenguang Liu ( $\square$ liweiguangl@126.com )

Jiangsu University

Haijiang Liu

Jiangsu University

lin Jiang

YAPP automotive systems Co.Itd.

Shanshan Bi

Jiangsu University

\section{Original Article}

Keywords: fuel tank, contact analysis, collision, defense point methoddeformation

Posted Date: September 9th, 2020

DOI: https://doi.org/10.21203/rs.3.rs-71519/v1

License: (c) (1) This work is licensed under a Creative Commons Attribution 4.0 International License. Read Full License 


\title{
Analysis of fuel tank collision structure based on defense point method
}

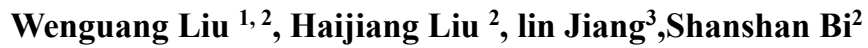 \\ 1.Tongji University \\ 2.School of Automotive and Traffic Engineering, Jiangsu University,301 Xuefu Road, Zhenjiang, Jiangsu Province, China. \\ 3.YAPP Automotive Systems Co, Ltd. \\ Corresponding Author: liuzhangwang2000@163.com; liweiguang1@126.com
}

Abstract Aiming at the impact process of a fuel tank, which is a transient energy conversion process, the material absorbs energy through deformation to analyze the mechanical properties of the fuel tank during the impact process. The defense node method is adopted to simulate the dynamic response of the fuel tank during impact. The results show that it can accurately evaluate the safety of the container.

Keyword: fuel tank, contact analysis, collision, defense point method, deformation

\section{Introduction}

The automobile fuel tank is the important component of the automobile fuel supply system. The country has strict requirements for its safety and environmental protection. To make full use of the limited mechanical space and adapt to various vehicle types, the shape of the automobile fuel tank is varied. The classical mechanic's method can not analyze and answer the fuel tank of various schemes at the same time, and there can be no measured data at the beginning of the design. Therefore, it is significant to check the strength and stiffness of the fuel tank effectively and quickly, and find out the weak links of the fuel tank, to provide a reference for the design and modification of the automobile fuel tank. At the same time, to shorten the development cycle, improve the quality of development, adapt to the rapid growth of the market requirements, and enhance the competitiveness of the market has important practical significance. However, the fuel tank manufacturer still stays at the stage of trial and error in the design of fuel tank structure, mainly relying on the experience of designers, hardly calculates and optimizes the shape of reinforcing ribs, the thickness of the tank wall, the aperture of the wave-proof plate and so on, which results in the unreasonable distribution of the material of the tank. The general design process is to manually make fuel tank product samples at first, and then to find out the problems by testing and modify them. From design to the factory, a product needs a long design cycle. Especially for the fuel tank, it has a large volume, complex structure, and many related inspection indicators, which leads to an increase in production costs.

The fuel tank impact test is a simulated impact test of the product. The purpose of this test is to verify whether the product or equipment is qualified in all aspects of the product when it subjected to external force shock or external force under normal operating conditions. Because collision is a complex process, it is affected by many factors, such as the constraints of the collision body, the relative speed of contact, the geometry and duration of the contact surface, local plastic deformation, etc. ${ }^{[1-3]}$ Aiming at the collision model of the system, Stronge analyzed the energy change during the oblique collision and the velocity relationship before and after the collision, and established the dynamic model of viscous/sliding friction contact ${ }^{[4]}$. The CEL method used to simulating the dynamic response of liquid storage vessel in the process of drop collision and the space motion state of liquid at different times. By comparing with the results of previous literature, the dynamic response and space state in the process of drop 
collision are discussed in four cases. Namely, different drop angle, drop height, vessel thickness, and liquid storage capacity, and the impact of factors on the vessel is also discussed ${ }^{[5]}$. The composite structure is vulnerable to all kinds of low energy impact during the process of production and application, which will make invisible visual damage in the laminates and degenerate the mechanical properties of the composite. Composite laminate is closely related to structural safety and life expectancy. So it is significant to evaluate the low-energy impact resistance and damage prediction of composite structures.

In a variety of collision detection algorithms, the oriented bounding box algorithm had widely used. By using the characteristics of the triangle surrounded by the rectangle in the leaf node and the value calculated in the rectangle-rectangle intersection test phase, the new algorithm contains a better triangle-triangle intersection algorithm in the oriented bounding box. In the original algorithm, to convert the two triangles into the same coordinate system before the two triangles were to test, but this step could omit by using the coordinates of the bounding boxes to replaces the coordinates of the triangles. This method reduces a lot of redundant coordinate transformation operations compared to the original algorithm ${ }^{[6]}$. Chai Lin established a single-degree-offreedom collision vibration model and studied the effects of parameters such as collision clearance, damping, stiffness, and excitation frequency on the bifurcation and chaos phenomena of the system motion using non-linear dynamic analysis and numerical simulation ${ }^{[7]}$. Fan Jianping uses the penalty stiffness method in the contact algorithm. By adjusting the penalty stiffness value reasonably, controlling the penetration distance effectively, and avoiding the ill-conditioned stiffness matrix, which makes the calculation result approach the true value ${ }^{[8]}$. Collision detection is a hot topic in computer graphics, augmented reality, humancomputer interaction, and other fields. In recent years, real-time simulation of large-scale complex scenes has attracted many scholars' attention, especially the emergence of cloud computing and big data technology, which puts forward higher requirements for real-time scene simulation, which also brings unprecedented opportunities and challenges to researchers. As the geometric complexity of the virtual environment increases, the computational complexity of collision detection greatly improved, and the interaction of complex scenes consumes a lot of computer resources. Therefore, the fast collision detection problem has become a bottleneck in the virtual environment. How to design an efficient collision detection algorithm to meet the requirements of realtime and accuracy has become a current problem to be solved. Qu had put forward a multiple date parallel collision algorithm based on optimization operator. The search space confined in a non-uniform local minimum area to reduce the colony search time. [9]. Chen Chengjun based on the local search algorithm of surface, characterized the contact sheet by the coordinates of the center of the face and the length of the feature, carried out pre-search to quickly eliminate potential contact pairs that would not occur contact, eliminated the blind area of contact search, and had good robustness and calculation accuracy ${ }^{[10]}$. Li Zhao proposes an improved collision detection algorithm based on deformable objects, which is difficult to solve the real-time and fidelity problems of deformable objects. To improve the efficiency of collision detection, an improved particle selection method and the idea of a multi-swarm particle optimization algorithm are used to construct a multi-line group on the multi-line composed of the control point cluster and the center point of the Snake model ${ }^{[1]]}$. Due to the short action time of external transient load and the difficulty of experiment control, the measured data are limited, and the continuous results in space and time do not obtain. Wang Bin simulated and analyzed the drop of metal cylinder structure under empty shell condition by ANSYS/LS-DYNA software, and studied the strain distribution law of metal thin-walled cylinder structure under different drop conditions (different height and different drop angle). The variation of stress and impact duration, impact force, and peak overload (impact acceleration) are discussed $^{[12]}$. An algorithm for simulating friction contact between soil and rigid or flexible structure in the SPH frame is proposed $^{[13]}$. The calculation domain divides into several subdomains, and the contact force is used as a bridge to establish the connection between the subdomains, to finally realize the global solution. When the SPH discretizes governing equations of soil motion in each subdomain, the inherent boundary defects of SPH are corrected. It makes the SPH particles near the contact boundary have accurate acceleration, which ensures the accuracy of contact detection. It assumed that the soil SPH particles are 
Normal and tangential contact forces of the contact surface are corrected by the slip condition so that they do not exceed the limit friction. Compared with the existing methods which usually use "particle-particle" contact or ignore friction slip in SPH, the method has higher calculation efficiency and accuracy. It is suitable for the simulation of the interaction between geotechnical materials and rigid or deformable structures. The accuracy and stability of this method verifies in many examples. The calculation shows that the SPH bases on a contact algorithm. The results are in good agreement with the theoretical solution or the finite element solution. The algorithm is effective and can be used to expand the calculation ability and application scope of the SPH. Considered the fluid-structure interaction effects in the analysis of liquid storage container dropping ${ }^{[14]}$, CEL method simulates the inertia effect of fluid and the lateral hydraulic pressure to the container, and the fluid-structure interaction effects on the deformation and dynamic response of the container during the process of dropping are considered. The numerical result shows that the method can provide a more accurate evaluation of vessel safety and structural design. Therefore, the CEL mentioned in the paper also provides a reference design evaluation method for the same structure. Considered particularity and uncertainty of drop impact crashworthiness design, the dynamic response of drop impact and impact crashworthiness robust design has been made. Drop impact crashworthiness robust design optimize frame present to base on crashworthiness evaluation for virtual drop test ${ }^{[15]}$. This work enriches the design theory and method for dynamic design. Precision electronic products mobile hard disk and liquid-sold coupled fluid-filled containers are chosen as two samples to investigate the capability of dynamic drop-impact, crashworthiness design, optimism of structural parameters, and design of crashworthiness.

The collision of the fuel tank is a transient energy conversion process in which the material absorbs energy through deformation. However, the structure of the fuel tank is sophisticated, which will lead to long calculation time and difficulty to ensure accuracy in collision operation. Aimed at the structural characteristics of the fuel tank, this paper adopts a defense node algorithm that avoids solving simultaneous equations and ensures constraints and accurately calculates contact force.

\section{Dynamic performance analysis of tank collision}

\subsection{Material collision mechanical properties}

For the collision body made of the fuel tank, the maximum shear stress $\sigma_{\tau}$ is related to the surface pressure. The following simple formula expresses their relationship ${ }^{[16]}$.

Among them, $v$ is the velocity at contact.

The maximum value of $\sigma_{\tau}$ occurs at $s \approx 2 /(1+v) \pi$ and $q_{0}(t)$ is the maximum surface pressure at given time t. For materials with low shear strength, collision will cause shear failure near the surface. The maximum value occurs at $0.5 t_{0}$, and the its duration occurs at $t_{0}$. The equivalent plastic strain criterion is used for the failure of fuel tank structural materials. When the equivalent plastic strain of the element reaches the threshold, the material is destroyed, and the corresponding part be deleted.

\subsection{Fluid-solid Coupling Analysis}

The dynamic characteristics of liquids are affected by the geometrical its, filling height, internal structure distribution, frequency, and amplitude of load excitation. At the same time, the physical properties of liquids such as density, compressibility, and viscous also have different effects on the dynamic characteristics.

The basic principle of the continuum equation is the conservation of mass. The mass $\delta m$ contained in the Lagrangian infinitesimal fluid unit $\delta V$ is $^{[17]}$ 
115 In the formula, $m$ is mass, $\rho$ is density.

116 For the mass conservation is satisfied in the Lagrangian fluid unit, the mass does not change with time, that is to say, the 117 following equation is satisfied.

$$
\frac{d \delta m}{d t}=\delta V \frac{d \rho}{d t}+\rho \frac{d \delta V}{d t}
$$

The fluid is a uniform, non-viscous, and incompressible theoretical fluid, ignored the fluid-solid momentum transfer and the local pressure-density linear relationship ${ }^{[18]}$. The coupling equation is:

$$
\left[\begin{array}{cc}
M_{S} & 0 \\
A^{T} & M_{f}
\end{array}\right]\left\{\begin{array}{l}
\ddot{u} \\
\ddot{p}
\end{array}\right\}+\left[\begin{array}{cc}
K_{S} & -A \\
A^{T} & K_{f}
\end{array}\right]\left\{\begin{array}{l}
u \\
p
\end{array}\right\}=\left\{\begin{array}{l}
F_{s} \\
F_{f}
\end{array}\right\}
$$

Where: $M_{s}$ and $K_{s}$ are the mass matrix and stiffness matrix of the structure; $M_{f}$ and $K_{f}$ are the mass matrix and stiffness matrix of the fluid respectively; $A$ is a fluid-solid coupling matrix; $F_{s}$ and $F_{f}$ are structural loads and acoustic loads, respectively; $u$ is the structural node displacement vector; $p$ is the fluid node pressure vector.

On the coupling interface $\Gamma$, the displacement and load balance conditions meet the following formula:

$$
\begin{aligned}
& d^{s} \cdot e^{s}+d^{f} \cdot e^{f}=0 \\
& \sigma^{s} \cdot e^{s}+\sigma^{f} \cdot e^{f}=0
\end{aligned}
$$

Where $d^{s}$ and $d^{f}$ are the displacement vectors of the solid domain and fluid domain interfaces, respectively. $\sigma^{s}$ and $\sigma^{f}$ are the stress vectors at the interface of the solid domain and the fluid domain, respectively. $e^{s}$ and $e^{f}$ are the unit displacement base vectors of the solid domain and the fluid domain interface, respectively.

\section{Contact analysis}

\subsection{Research on contact problems during collision contact}

The collision process of the fuel tank has nonlinear characteristics. When the collision height is short, and the impact energy is small, the elastic force $F(x)$ of the cushioning material has a linear relationship with the deformation $x$. That is to say; Simplified the buffer material becomes a single-degree-of-freedom spring-mass system with a constant stiffness coefficient in the process of drop impact.

The dynamic impact process is a series of dynamic processes varying with time. Subjected to shock excitation, the system will produce a corresponding shock response. It proves theoretically that the maximum value is related to the duration of shock $\tau$ and the inherent period $T_{n}$ of the system itself after shock excitation. When $T_{n}<\tau$, the maximum shock response of the system may be twice the peak value of the shock wave, while when $T_{n}>\tau$, the shock response will be weakened. The impact process is a transient energy conversion process, and the buffer material absorbs energy through deformation.

(1) Kinematic constraints

The contact and collision structure of the fuel tank shows in Fig. 1. When two contacts are in contact, two points $x_{2}^{t}$ and $x_{1}^{t}$ coincide at the contact interface. Point $x_{2}^{t}$ is the orthogonal projection of point $x_{1}^{t}$ on boundary $\Gamma^{1}$ of contact 1 on boundary $\Gamma^{2}$. According to kinematic constraints, the formula is as follows: ${ }^{[19]}$ 
Among them, $e_{1}$ is the usual unit vector.

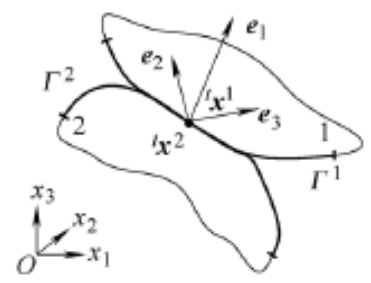

Fig. 1 Contact-impact interface model

(2) Dynamic constraints

The contact force on the unit contact surface is pressure, and its pressure value $p_{e}$ should be satisfied.

$$
p_{e}>0
$$

The tangential friction $p_{e t}$ on the contact surface is the resultant force of the tangential contact forces in the other two directions.

$$
p_{e t}=\sqrt{p_{e t 2}^{2}+p_{e t 3}^{2}}
$$

Among them, $p_{e t 2}$ and $p_{e t 3}$ are the tangential friction forces of unit tangent vectors $e_{2}$ and $e_{3}$, respectively.

If the Coulomb friction model is adopted, when the maximum static friction force is less than the maximum static friction force, the points on the two contact surfaces are relatively static, that is, the relative tangential velocity A of the points on the two contact faces is

$$
v_{t}^{t}=\left(v_{1}^{t}-v_{2}^{t}\right)-\left[\left(v_{1}^{t}-v_{2}^{t}\right) \cdot e_{1}\right] e_{1}=0
$$

Among them, $v_{1}^{t}$ and $v_{2}^{t}$ are the tangential relative speeds of points $x_{1}^{t}$ and $x_{2}^{t}$, respectively.

When it is equal to the sliding friction, point $x_{1}^{t}$ and point $x_{2}^{t}$ are relatively slipped

$$
v_{t}^{t} \cdot\left[p_{1, c}^{t}-p_{1, c}^{t} \cdot e_{1}\right] e_{1} \leq 0
$$

Because the collision time is short and the tangential force is relatively small relative to the normal force, ignored the influence of the tangential force during the collision.

\subsection{Contact force algorithm for contact collision}

Found out the contact point after the analysis of the contact interface, and then calculated the contact force using the motion law of the object. Limited the value of the contact force contact constraints. The contact point is not allowed to penetrate the contact boundary, and the contact force cannot be a tensile.

The usual methods to calculate contact force are the penalty function method and the Lagrange multiplier method. These two methods are quite different in the explicit algorithms and the implicit algorithms. Lagrange multiplier method involves solving the simultaneous equations of unknown contact forces, which can not be directly used in the explicit algorithm, in contrast it does not solve any simultaneous equations. Therefore, in the explicit algorithm, the penalty function method is often used to calculate the contact force. 
The penalty function method has the disadvantages of introducing artificial error and affecting the stability of the explicit algorithm. To make the error introduced by penalty function method small enough, choose reasonable penalty parameters, effectively control the stability of it, and select appropriate penalty parameters, is the most significant problem of using penalty function method to calculate contact force. Another one is the calculation of contact penetration. To avoid the error caused by the penalty function method and the influence on the stability of the solution, some particular algorithms are used to solve the contact force by the Lagrange multiplier method, which avoid solution of the simultaneous equations.

For implicit algorithm, whether the penalty function method or Lagrange multiplier method is used, it involves the establishment of contact stiffness matrix, and different iterative algorithms have different requirements for contact stiffness matrix. In the implicit algorithm, a new contact point may not only affect the number of zero elements in the coefficient matrix of the algebraic equation but also affect the bandwidth of the coefficient matrix. When used the Lagrange multiplier method, the total number of unknowns increases, which leads to the change of dimension of the coefficient matrix. So these will affect the memory allocation and management in the process of solving, and also affect the calculation workload. In the implicit algorithm, if the penalty function method is used to calculate the contact force, the penalty parameter should not be too large, otherwise, the coefficient matrix may become ill-conditioned and hinder the solution.

The contact algorithm accomplishes the interaction between colliding structures or components. Within each time step of the solution, checked whether the slave node penetrates the main surface. If it penetrates, the force is applied in a direction perpendicular to the main surface by a penalty function to prevent further penetration of the slave node, if not worn. Pass through, then proceed. In the penalty function method, it is difficult to select the penalty parameter value, and it is difficult to obtain the ideal calculation result by experience. To ensure accuracy, the defense node method is used to calculate the contact force. That is, the Lagrange multiplier method is used to satisfy the constraints accurately, and the algorithm of solving simultaneous equations is avoided. The defense node is calculated by adding a virtual contact point to each contact pair.

Assuming the mass of the defense node is $\mathrm{M}$, the motion equation of the defense node and the contact point can be written as follows ${ }^{[20]}$

$$
\begin{aligned}
& M_{1} a_{1}=F_{1}+f_{1} \\
& M_{2} a_{2}=F_{2}+f_{2}
\end{aligned}
$$

Among them, subscripts 1 and 2 represent respectively from the contact point and the defense node. $F$ and $f$ are normal nodal force and contact force, respectively.

Used to the Lagrange multiplier method calculates the contact force to satisfy the constraints. The motion equation is obtained by using the central difference method from the contact point and the defense node.

$$
\begin{array}{r}
M_{1}\left[{ }^{\tau} v_{1}-\left({ }^{t} s_{1}-{ }^{\tau} s_{1}\right) / \Delta t\right] / \Delta t={ }^{\tau} F_{1}+{ }^{\tau} f_{1} \\
M_{2}\left[{ }^{\tau} v_{2}-\left({ }^{t} s_{2}-{ }^{\tau} S_{2}\right) / \Delta t\right] / \Delta t={ }^{\tau} F_{2}+{ }^{\tau} f_{2}
\end{array}
$$

Among them, ${ }^{\tau} S,{ }^{\tau} v$ are the displacement and velocity after contact, ${ }^{t} s,{ }^{t} v$ are the displacement and velocity before contact.

According to the same magnitude of the defense point force and the force from the contact point, the normal distance is 0 , and the defense node force is 0 .

$$
{ }^{\tau} f_{1}=M_{1} M_{2}\left({ }^{\tau} F_{2} / M_{2}-{ }^{\tau} F_{1} / M_{1}+{ }^{l} v_{2} / \Delta t-{ }^{l} v_{1} / \Delta t-{ }^{\tau} g / \Delta t\right) /\left(M_{1}+M_{2}\right)
$$


Among them, ${ }^{\tau} g$ is the total gap between the defensive point and the contact point.

The contact search algorithm plays an significant role in reducing calculation time and improving calculation accuracy. Any contact level has its contact domain, which can be defined as an extension domain. If a slave falls into an extension domain of the main block, the two may contact to form a test pair. If a slave falls into the contact area of it (edge, point), the two contact and shape a contact pair.

Contact search includes pre-contact search and post-contact search. The pre-contact search is usually divided into two steps, i.e., global search and local search, for the slave points that are not in contact state in the previous calculation. The global search roughly determines the slave points that may be contacted, and the main blocks that may be contacted with the slave points processed, which is to find out all the test pairs. Local search accurately locates the target of the slave point, calculate the penetration of the slave point relative to its target point, and judge the contact state of the slave point, to find out all contact pairs from the test pair.

Through the contact search algorithm, all the slave points in contact state and their corresponding main blocks (edges, points) in the mechanical system are determined, and the homologous contact force algorithm is used to calculate the contact force. Used the explicit calculation of the central differential, and used the contact algorithm to solve the problem. Then the acceleration of the nodes is used to calculate other physical quantities.

\section{3Stress Failure Criteria}

The surface pressure caused by collision, the failure form caused by three-dimensional internal stress, and the time sequence of various failure forms. Therefore, appropriate failure criteria can be adopted for the three-dimensional stress state caused by a collision at each point in the collision object. For composite materials, there are three common criteria:

Cai-Hill Tsai-Hill Strength Theory

$$
\frac{\sigma_{L}^{2}}{F_{L}^{2}}-\frac{\sigma_{L} \sigma_{T}}{F_{L}^{2}}+\frac{\sigma_{L}^{2}}{F_{L}^{2}}+\frac{\tau_{L T}^{2}}{F_{L T}^{2}}=1
$$

$\frac{\sigma_{L}^{2}-\sigma_{L} \sigma_{T}}{F_{L t} F_{L c}}+\frac{\sigma_{L}^{2}}{F_{L}^{2}}-\frac{F_{L c}-F_{L t}}{F_{L t} F_{L c}} \sigma_{L}+\frac{F_{T c}-F_{T L}}{F_{T L} F_{T c}} \sigma_{T}+\frac{\tau_{L T}^{2}}{F_{L T}^{2}}=1$

$\frac{\sigma_{1}^{2}}{F_{L t} F_{L c}}-\frac{\sigma_{1} \sigma_{2}}{\sqrt{F_{L t} F_{L c} F_{T t} F_{T c}}}+\frac{\sigma_{2}^{2}}{F_{L t} F_{L c}}+\frac{\sigma_{6}^{2}}{\tau^{2}}+\frac{F_{L c}-F_{L t}}{F_{L t} F_{L c}} \sigma_{1}$

$+\frac{F_{T c}-F_{T t}}{F_{T} F_{T t}} \sigma_{2}=1$

For the plane stress-strain state, the improved maximum stress failure criterion is used to judge the failure of the matrix material.

\section{Analysis of simulation results}

The fuel tank used in this calculation shows in Fig. 2. According to the requirements of the enterprise, used $348 \mathrm{~kg}$ slider impact 

results show in the figure.

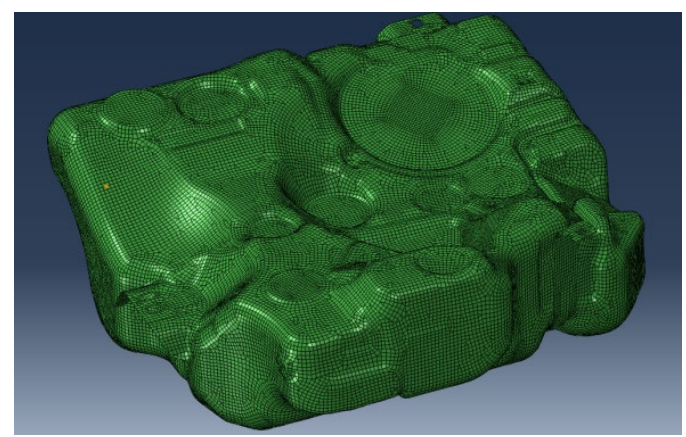

Fig. 2 Fuel tank structure diagram

247 in a different direction, the major deformation shows in Figure 3.

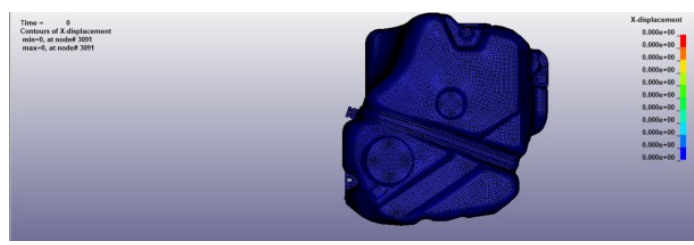

(a) Before the collision

(b) Displacement diagram in $\mathrm{X}$ direction after the collision
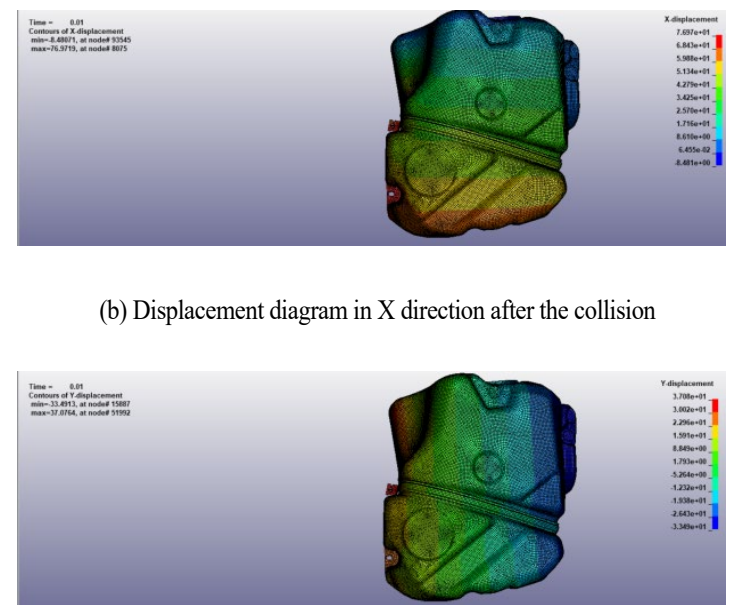

(c) Displacement diagram in Y direction after the collision

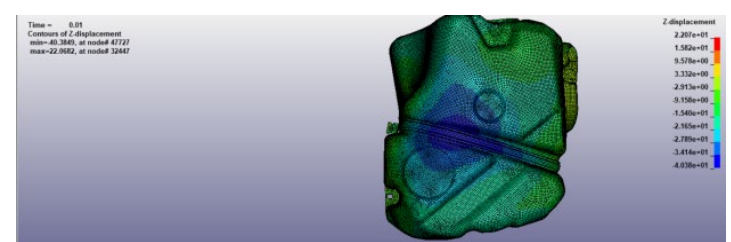

(d) Displacement diagram in Z direction after the collision

Fig. 3 Displacement diagram of the Fuel tank

257 In terms of displacement, the front end with relatively large deformation is taken. As the fuel tank is a curved part, it is 
convenient to fix the sensor. The sensor is placed on the plane of the fuel tank in the front section. After measuring its position, the collision and simulation experiments are carried out (fig. 4). In the process of impact test, the major deformation is in the $\mathrm{x}$ axis direction, so this test also mainly considers the impact on the $\mathrm{x}$-axis, and the simulation results are consistent with the experimental results, meeting our impact requirements.

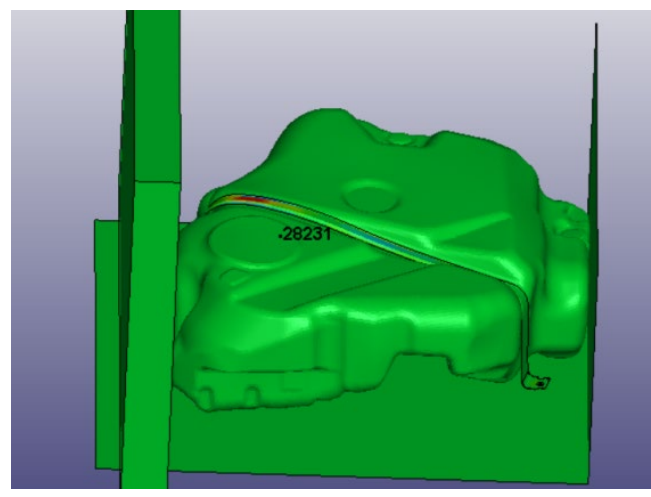

(a) Test point

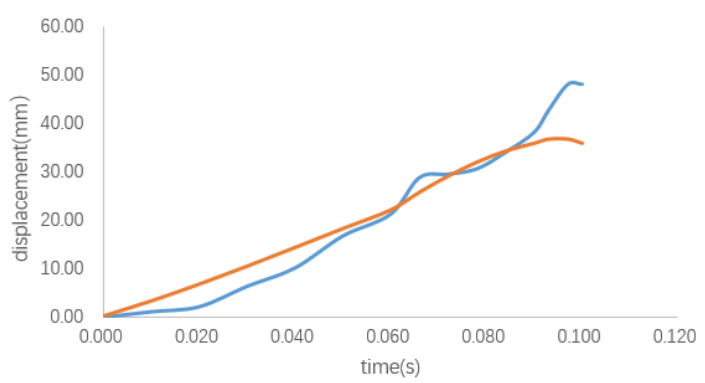

(b) X displacement of test point

\section{CONCLUSION}

In this paper, the structure of the fuel tank is analyzed, and the mechanical analysis of the collision body made of the fuel tank is carried out. In the collision operation, the problems such as long calculation time and difficult to guarantee accuracy are caused. The collision impact process of the fuel tank is analyzed, and the deformation process of the fuel tank is simulated and analyzed by the defense node algorithm. This study has important practical significance for the actual collision process of the fuel tank.

\section{Declarations}

\section{Acknowledgements}

\section{$274 \quad$ Funding}

275 This work was supported in part by a grant from the Jiangsu Provincial Six Talent Projects Funding (JXQC-009).

\section{Authors' contributions}

277 Wenguang Liu: analysis;supervision

278 Haijiang Liu:analysis

279 lin Jiang:analysis 
281 Supplemental Instruction

282 This paper has not a conflict of interest statement.

Data Availability Statement

The data that support the findings of this study are available from the corresponding author upon reasonable request.

\section{REFERENCE}

[1] Gao Yang, Ge Zhishang, Zhai Weihao, et al. The Finite Element Modelling and Dynamic Characteristics Analysis about One Kind of Armoured Vehicles' Fuel Tanks[C]// Materials Science \& Engineering Conference Series. Materials Science and Engineering Conference Series, 2018.

[2] Magdum Supriya J, Sivakumar Ramasami, Sakthivel G, et al. DOE study of 'effect of various parameters on fuel tank sloshing using multiphase CFD'[J]. International Journal of Ambient Energy, 2018:1-23.

[3] Mocilan Martin, Zmindak, Milan, Pastorek Pastorek. Dynamic Analysis of Fuel Tank[J]. Procedia Engineering, 2016, 136:45-49.

[4] W. J. Stronge, R. James, B. Ravani. Oblique impact with friction and tangential compliance[J]. Philosophical Transactions Mathematical Physical \& Engineering Sciences, 2001, 359(1789):2447-2465.

[5] Zhang Liu Hao. Finite Element Simulation on Dropping Impact of Liquid Storage Container[D]. DALIAN UNIVERSITY OF TECHNOLOGY,2014

[6] Liu Chao, Jiang Xiajun, Shi Huibin. Mended collision detection algorithm based on oriented bounding box[J]. Computer Technology and Development. 2018, v.28; No.254(06):49-54.

[7] Chai Lin, Wu Xiao-ming. Evolution of Bifurcation and Chaos in Mechanical Vibro-impact System with Parameters[J]. Journal of Xiamen University (Natural Science), 2014, 53(4):508-513.

[8] Fan Jiaping, Wu Mengchang, Hu Jun, etc. Dynamic Analysis of Vehicle-bride Interaction Based on Interfacial Contact Algorithm[J]. China Journal of Highway and Transport, 2017, 30(4):52-58.

[9] Qu Huiyan, Zhao Wei, Qin Aihong. A fast collision detection algorithm based on optimization operator[J]. Journal of Jilin University(Engineering and Technology Edition) 2017, 47(5):1598-1603.

[10] Chen Chengjun, Liu Ming, Chen Xiaowei, etc. A contact-impact algorithm based on the segment-to-segment local searching method[J]. Chinese Journal of Computational Mechanics, 2018(1):105-110.

[11] Li Zhao, Jin Yanxia, Qin Zhipeng, etc. Collision Detection Algorithm of Deformable Object-Based on Snake Model Optimization[J]. Journal of System Simulation, 2018(1):62-68.

[12] Wang Bin. Metal thin-walled tube structure drop experiment and simulation[D]. Beijing Institute of Technology, 2015

[13] Wang Jian, Wu Hao, Gu Chongshi, etc. Simulation of friction contact in smooth particle hydrodynamics (SPH) [J]. China Science: technology science, 2013 (11): 1208-1218

[14] Nie Junfeng, Zhang Haiquan, Li Hongke, etc. Dropping Accident Analysis of Liquid Storage Container using Finite Element 
314 [15] Yang Shuyi. Drop Impact Dynamics and Crashworthiness Robust Design for Manufactured Products[D]. China University of Mining and technology. 2009.

316 [16] Zhang Zhen. A study on impact of composite material CNG cylinder[D]. North China University Technology, 2006

317 [17] Liu Fu. Dynamic Analysis of Liquid Sloshing and Sloshing Suppression Design for a Tank[D]. Nanjing University of Aeronautics 318 and Astronautics, 2010

319 [18] Fu Guang, Liang Jingqiang, Luo Huijuan, etc. Fluid-Structure coupling Modal Analysis of Auto oiltank[J]. Automotive Technology, 2016(2):25-28.

[19] Zhong Yang, Zhong Zhihua, Li Guangyao, etc. Review on Contact Algorithms Calculating the Contact-impact Interface in Mechanical System with Explicit FEM[J]. Journal of Mechanical Engineering, 2011, 47(13):44-58.

[20] Hong Haijin, Li Guangyao. Analysis of Sheet Metal Forming Based on Defence Node Algorithm[J]. Computer Simulation, 2011, 28(8):320-322. 
Figures

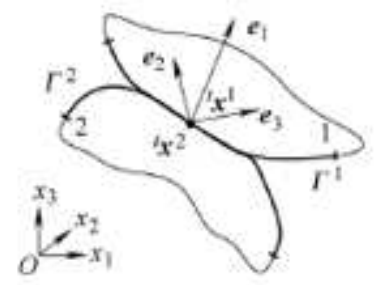

Figure 1

Contact-impact interface model

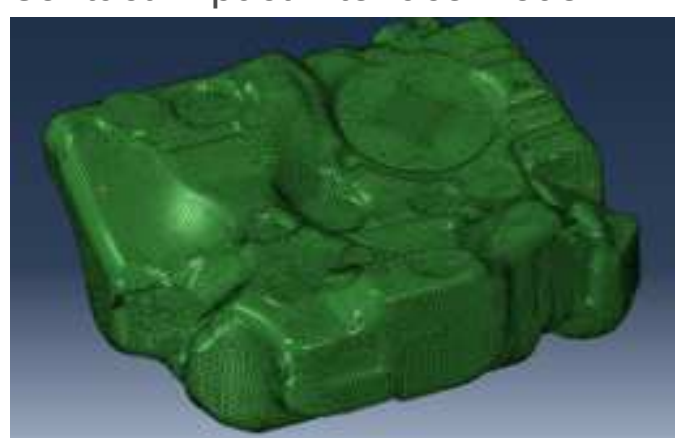

Figure 2

Fuel tank structure diagram 


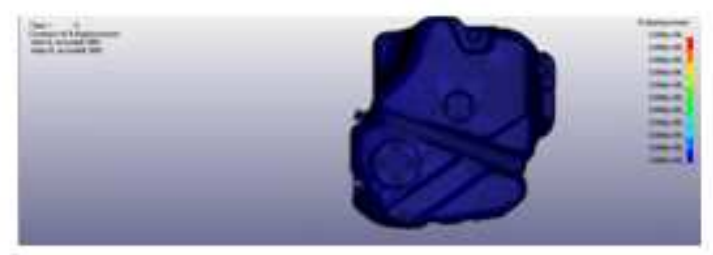

(a) Before the collision

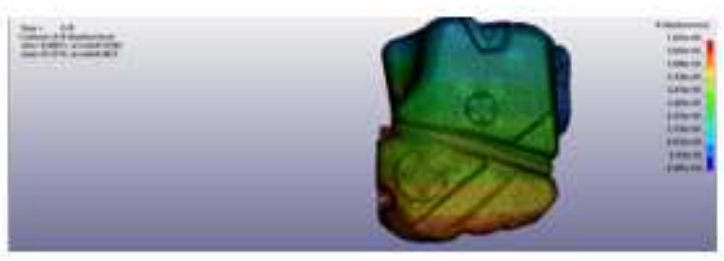

(b) Displacement diagram in $\mathrm{X}$ direction after the collision

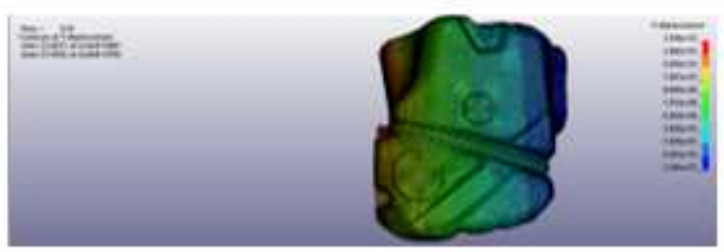

(c) Displacement diagram in $\mathrm{Y}$ direction after the collision

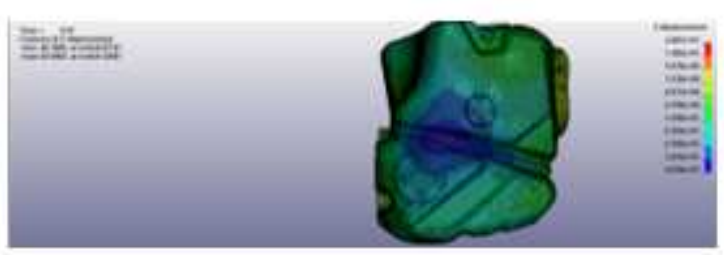

(d) Displacem ent diagram in $\mathrm{Z}$ direction after the collision

Figure 3

Displacement diagram of the Fuel tank 


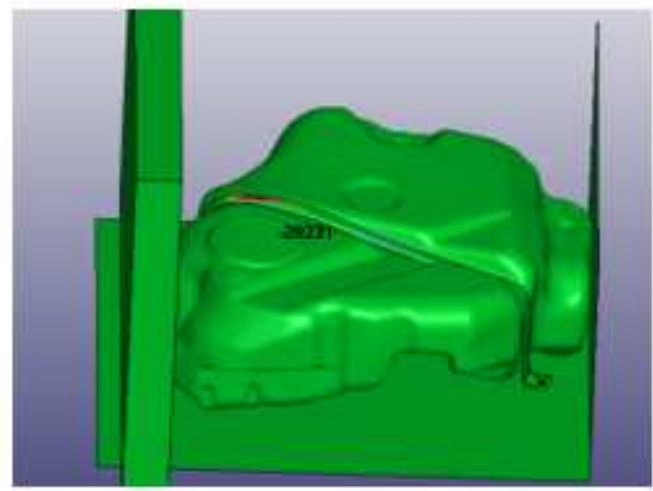

(a) Test point

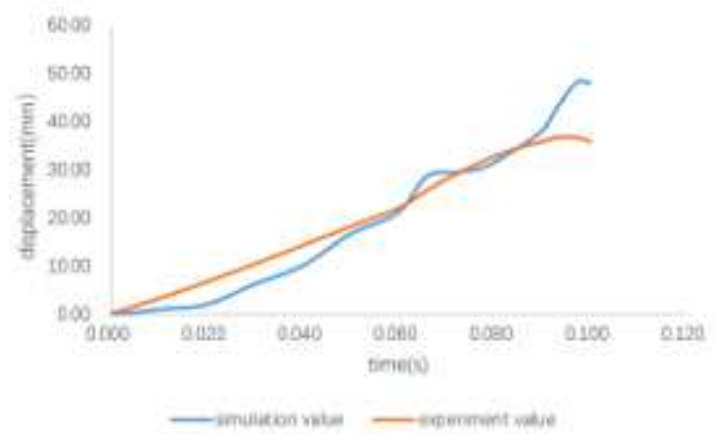

(b) X displacement of test point

\section{Figure 4}

Fuel tank configuration and stress-strain diagram 